\title{
Au cœur d'un quartier de Condate. La fouille archéologique de l'ancien hôpital militaire Ambroise-Paré de Rennes
}

Nicolas Mathieu

\section{(2) OpenEdition \\ Journals}

Édition électronique

URL : https://journals.openedition.org/abpo/6543

DOI : $10.4000 / a b p o .6543$

ISSN : 2108-6443

Éditeur

Presses universitaires de Rennes

\section{Édition imprimée}

Date de publication : 10 décembre 2020

Pagination : 205-207

ISBN : 978-2-7535-8225-5

ISSN : 0399-0826

Référence électronique

Nicolas Mathieu, «Au cœur d'un quartier de Condate. La fouille archéologique de l'ancien hôpital

militaire Ambroise-Paré de Rennes ", Annales de Bretagne et des Pays de l'Ouest [En ligne], 127-4 | 2020, mis en ligne le 10 décembre 2020, consulté le 05 janvier 2023. URL : http://journals.openedition.org/ abpo/6543 ; DOI : https://doi.org/10.4000/abpo.6543 


\section{Comptes-rendus}

LE CloIREC, Gaétan (dir.), Au coeur d'un quartier de Condate. La fouille archéologique de l'ancien hôpital militaire Ambroise-Paré de Rennes, Rennes, PUR, 2020, 268 p., ill. N.B. et couleurs, biblio. ISBN 978-2-7535-8024-4. $39 €$.

La fouille archéologique de l'ancien hôpital Ambroise-Paré de Rennes, dirigée par G. Le Cloirec, s'est déroulée en 1999-2000, dans un terrain de $2000 \mathrm{~m}^{2}$ situé au centre-ouest de l'emprise de la ville du Haut-Empire et en dehors de la ville ceinte d'une muraille au Bas-Empire, sur une terrasse fluviale de rive gauche de la Vilaine, dans un secteur qui a été peu occupé aux époques médiévale et moderne. L'ouvrage qui en résulte, dirigé G. Le Cloirec, est judicieusement titré $\mathrm{Au}$ coeur d'un quartier de Condate. En effet, cette fouille a eu le double mérite, par l'ampleur de la superficie explorée, non seulement de fournir beaucoup d'éléments d'informations, mobiliers et immobiliers, calés dans une longue durée, mais aussi de pouvoir corriger, compléter, enrichir les connaissances très ponctuelles et localisées d'une douzaine de fouilles antérieures, depuis la décennie 1970 tout autour dans un rayon de 100 à 150 m (figure 8, p. 15). De la sorte, elle offre comme une mise en perspective sur tout un secteur de la ville jusqu'alors partiellement connu par des coups de projecteurs sans continuité spatiale assurée.

Ouvrage de synthèse, ce livre est nourri des contributions des nombreux spécialistes qui ont participé à la fouille ou ont été sollicités pour interpréter la documentation mise au jour : Françoise Labaune-Jean, Stéphane Jean, Paul-André Besombes, Stéphan Hinguant, Anne Gebhardt, Jean-Pierre Bost, Georges Fabre, Yvan Maligorne, Dominique Marguerie, Vincent Bernard, Yannick Le Digol, Véronique Guitton.

Le livre est organisé en trois chapitres et suivi d'une conclusion, p. 245-246, et de la bibliographie, p. 247-253. Il est illustré de nombreuses figures (213 numéros) consistant en photographies en N.B. et en couleurs, plans, coupes, dessins, et de tableaux (35 numéros) sur le mobilier céramique et les monnaies principalement. C'est un beau travail d'édition dans tous les sens (scientifique, de composition). La lecture est agréable, le style fluide, la langue aussi précise que simple. Quelques " au final " ou " suite à " sont à déplorer et un " bien que " suivi d'un imparfait de l'indicatif (p. 14).

Le premier chapitre, dû à G. Le Cloirec et St. Hinguant, « Du projet immobilier à la fouille ", p. 9-20, expose le cadre topographique, historiographique et historique de la fouille. Il en ressort une confirmation du développement de l'agglomération antique sur le versant occidental de la colline Saint-Melaine vers la rive gauche de l'Ille; une présence d'abord d'activités artisanales, identifiées par de nombreux fours de potiers alors que plus au nord, dans le secteur du cardo de la rue de SaintMalo, connu par d'autres fouilles, les fours étaient ceux d'orfèvres ou de bronziers; un maintien durable d'une activité économique mais une réorientation de l'occupation devenue résidentielle ensuite.

Dans le deuxième chapitre, "Émergence et développement d'une parcelle d'histoire ", p. 21-137, vient la présentation de l'opération archéologique, secteur 
par secteur et élément stratigraphique par élément stratigraphique avec de nombreuses illustrations, coupes et plans toujours à propos au plus près du texte. Il est l'œuvre de l'ensemble des contributeurs puisqu'il s'agit de la description archéologique systématique de la fouille. Il ravira les archéologues et spécialistes mais ne sera peut-être pas lu comme un roman. Ce matériau est présenté avec beaucoup de clarté et de rigueur. On apprécie d'être pris en main, pas à pas avec des illustrations choisies, parfois surlignées de traits qui permettent de visualiser et concevoir un plan sur un vestige de mur arasé. On apprécie aussi qu'au terme de la présentation de chaque unité décrite une proposition de datation soit esquissée. Notons dans ce chapitre l'analyse (p. 50), par Y. Maligorne, spécialiste des éléments d'architecture notamment de l'Ouest armoricain (L'architecture romaine dans l'ouest de la Gaule, Rennes, PUR, 2006) d'un fragment de corne d'abaque d'un chapiteau corinthien ou composite avec un rang d'oves et de fers de lance qui pourrait mettre sur la voie d'un édifice public, peut-être cultuel (temple? mausolée?), qui pourrait être daté des julio-claudiens (Voir une première présentation de ce fragment d'abaque dans Aremorica, 9, 2018, p. 110-11 avec fig. 4). Jusqu'alors seuls deux fragments de chapiteaux corinthiens datés de la même époque avaient été trouvés, en remploi dans les fondations de la muraille.

Une fois établi ce corpus archéologique arrive le dernier chapitre (G. Le Cloirec, Fr. Labaune-Jean, P.-A. Besombes, J.-P. Bost et G. Fabre) « Agencement et occupation d'un îlot urbain à Condate ", p. 139-244, qui consiste en l'analyse et l'interprétation des données de la fouille (p. 139-244). C'est la synthèse historique : dès le début du principat le secteur a été occupé comme en témoignent les vestiges de quatre fours de potiers. Les structures construites montrent aussi une intégration à une organisation viaire planifiée mais non encore totalement réalisée, en tout cas avec des rues réduites à un simple lit de gravillons, et une absence d'habitations construites à l'époque initiale. Le secteur est alors en périphérie du noyau urbain. C'est à partir du milieu du $\mathrm{I}^{\mathrm{er}}$ siècle apr. J.-C., à l'époque claudienne, que sont probablement mises en œuvre les chaussées construites, point de départ du développement de l'urbanisme, qu'apparaissent les constructions maçonnées et, au $\mathrm{II}^{\mathrm{e}}$ siècle, que peuvent être identifiés des bâtiments plus imposants pour des usages qui excluent les activités marchandes entraînant des nuisances du type feux, fumées, bruits du travail. L'usage est d'habitation privée ou collectif ou communautaire. Le quartier a ainsi été occupé par plusieurs ensembles architecturaux significatifs : un bâtiment mixte à vocation domestique et artisanale, une domus à péristyle, un bâtiment communautaire, mansio ou siège de corporation. C'est de ces deux derniers bâtiments que proviennent les enduits peints qui appartiennent au groupe du troisième style pompéien (fin du $\mathrm{I}^{\mathrm{er}}$ siècle av. J.-C.- première moitié du $\mathrm{I}^{\mathrm{er}}$ siècle apr. J.-C.) et ceux qui ont été graffités. D'autres décors permettent d'envisager une datation jusqu'au milieu du II ${ }^{\mathrm{e}}$ siècle apr. J.-C.

Deux dossiers de matériel découvert à l'occasion de cette fouille ont nécessité l'aide de spécialistes en épigraphie et en numismatique : p. 178-183, J.-P. Bost et G. Fabre pour les graffiti découverts sur les nombreux fragments de peintures murales datées de la fin du $\mathrm{II}^{\mathrm{e}}$ et du $\mathrm{III}^{\mathrm{e}}$ siècle, provenant des vestiges du péristyle d'une domus. La petite série de graffites $\left(17 \mathrm{n}^{\text {os }}\right.$ au catalogue p. 178-182) fournit des noms connus ou nouveau, entiers ou lacunaires qui augmentent le stock onomastique dans la cité : Matto, peut-être Arugus, Macalus, Scita, et donne des exemples d'abécédaires ou de vantardises ou d'injures sexuelles. Quelle que soit leur nature, ces graffiti illustrent l'usage bien répandu de l'écriture. Il est très probable qu'ici, comme ailleurs, les alphabets, les répétitions de lettres témoignent de l'apprentissage de la lecture, de l'écriture tant par des enfants que par des adultes. Certains avaient déjà été présentés dans des articles peu diffusés (Voir $A E, 2008,907 \mathrm{a}, 908)$ ). P. 183- 
192, P.-A. Besombes étudie les quarante-et-une pièces différentes s'échelonnant du début du principat augustéen à la fin du $\mathrm{III}^{\mathrm{e}}$ siècle (Tétricus 1) qui ont été mises au jour, issues d'ateliers divers (Lyon, Rome, gaulois, Trèves, clandestins), avec une surreprésentation des monnaies à forte valeur (les quatre deniers équivalent à $16 \%$ des monnaies du Haut-Empire). Entre autres interprétations, il y a la possibilité que le grand nombre de monnaies d'époque augustéenne et julio-claudienne illustre la résidence sur place, la réalité d'une habitation et d'une activité au moins jusqu'au début des années 360 et l'abandon au IV siècle.

Le mobilier céramique abondant et les figurines en terre cuite, concentrées du ${ }^{\mathrm{er}}$ au milieu du $\mathrm{II}^{\mathrm{e}}$ siècle (études de F. Labaune-Jean), les objets en bronze, principalement des fibules, les monnaies, les graffites confirment l'intégration dans les circuits commerciaux et l'implantation à proximité des voies d'échange. Tout au long de l'analyse et de l'interprétation les auteurs mettent en perspective les documents, changent d'échelles et font des comparaisons avec l'ouest armoricain et, en matière architecturale, non seulement l'ouest et nord-ouest de la Gaule mais aussi l'Italie, notamment la Campanie et Pompéi. On ne mentionne que deux exemples : celui de l'organisation spatiale et de la trame urbaine (p. 139-148). Ici on trouve un tableau récapitulatif des chaussées urbaines des chefs-lieux des cités de l'Ouest armoricain connus en fouilles ou par des sondages, avec leur orientation, leur emprise au sol indiquée en pieds romains, et leur chronologie - dates de mise en place et d'abandon -; là des mises au point sur les types de maisons : en termes de conception du plan et de matériaux et structure, pour la maison de taille moyenne située dans la partie orientale du site (p. 148-158), et de même pour la domus à péristyle (p. 158-161). Ce sont les caractéristiques de leurs plans qui, par comparaison, conduisent à identifier un moment de l'histoire de la première avec une mansio, un macellum ou un siège de corporation (p. 156-157). Quant à la domus, les comparaisons avec d'autres constructions dans la province d'Aquitaine, chez les Lémovices à Limoges, ou à Pompéi, permettent de proposer une restitution des volumes (p. 159-161 avec la collaboration de V. Mutarelli). L'analyse ensuite du décor complète le tableau en insérant cet édifice dans l'épaisseur sociale.

Qu'il ait fallu attendre presque deux décennies la publication n'est guère étonnant dans les conditions actuelles du travail en archéologie. On ne s'en plaindra pas. Cela a permis de publier une synthèse de qualité, de l'insérer dans un champ de recherche plus large. La collection "Archéologie \& Cultures " s'enrichit ainsi d'un nouveau volume sur Rennes, douze ans après le livre dirigé par Dominique Pouille, Rennes antique (2008), vingt ans après le catalogue de l'exposition Fondations. Rennes et son pays dans l'Antiquité, dirigé par F. Hubert et Françoise Berretrot, et presque en même temps que d'autres publications : celle de l'Inrap, D. Pouille, Rennes antique vue par l'archéologie préventive (Paris, Rennes, Inrap, Musée de Bretagne, 2018, collection Mémoires de fouilles, 38, accessible en ligne en PDF); celle M. Six et A. Delécolle (dir.), Les vies d'une ville (Rennes, PUR, 2018) qui accompagnait une exposition au Musée de Bretagne. La fouille archéologique de l'ancien hôpital Ambroise-Paré est un élément supplémentaire dans le panorama du cheflieu des Riédons, Condate, mais aussi une monographie qui doit servir de comparaison avec d'autres cités provinciales. Point d'aboutissement d'une fouille un peu exceptionnelle par les dimensions du site, ce livre doit désormais être point de départ pour qui s'intéresse à l'urbanisme provincial, aux étapes de la mise en place de l'urbanisation avec un parcellaire caractéristique au i ${ }^{\mathrm{er}}$ siècle sous le principat augustéen et à l'époque julio-claudienne, d'abord à l'échelle de l'ouest armoricain dans la province de Lyonnaise puis au-delà.

Nicolas MATHIEU 